\title{
The prognostic role of ovarian endometriosis in symptomatic adenomyosis patients underwent uterine artery embolization
}

\author{
Wenbo Guo ${ }^{1 \#}$, Siqi Hu ${ }^{1 \#}$, Dan Zeng ${ }^{2}$, Chaogui Yan $^{3}$, Rui Zheng ${ }^{4}$, Jian Gao ${ }^{5}$, Tingting Wan ${ }^{6}$, \\ Wenquan Zhuang ${ }^{1}$, Jianyong Yang ${ }^{1}$
}

${ }^{1}$ Department of Interventional Radiology, the First Affiliated Hospital of Sun Yat-sen University, Guangzhou, China; ${ }^{2}$ Department of Ultrasound, the Eastern Hospital of the First Affiliated Hospital, Sun Yat-sen University, Guangzhou, China; ${ }^{3}$ Diagnostic Radiology, The First Affiliated Hospital of Sun Yat-sen University, Guangzhou, China; ${ }^{4}$ Department of Interventional Radiology, Tongling People's Hospital, Tongling, China; ${ }^{5}$ Department of Interventional Radiology, Baoan District People's Hospital of Shenzhen, Shenzhen, China; ${ }^{6}$ Diagnostic Radiology, Jiangxi Cancer Hospital; Nanchang, China

Contributions: (I) Conception and design: W Guo; (II) Administrative support: W Guo, J Yang, W Zhuang; (III) Provision of study materials or patients: D Zeng, C Yan; (IV) Collection and assembly of data: S Hu, R Zheng, J Gao; (V) Data analysis and interpretation: S Hu, T Wan; (VI) Manuscript writing: All authors; (VII) Final approval of manuscript: All authors.

\#These authors contributed equally to this work.

Correspondence to: Wenbo Guo. The First Affiliated Hospital of Sun Yat-sen University, Department of Interventional Radiology, No. 58 Zhongshan 2 Road, Guangzhou 510080, China. Email: guowenbo@mail.sysu.edu.cn.

\begin{abstract}
Background: To explore the prognostic role of ovarian endometriosis in symptomatic adenomyosis patients underwent uterine artery embolization (UAE).

Methods: This was a retrospective, single-center study. A total of 76 patients with adenomyosis who underwent UAE in The First Affiliated Hospital of Sun Yat-sen University between May 2009 and July 2016 were enrolled in this study. These patients were divided into two groups based on whether complicated with ovarian endometriosis. After UAE, the patients were followed up for 12 months. The improvements of dysmenorrhea and menorrhagia were evaluated according to the symptom relief criteria. The improvement rates in both groups were analyzed and compared.

Results: Among the 76 patients with adenomyosis, 17 (22.3\%) were diagnosed with OE and 59 (77.6\%) were non-OE. In the OE group, all patients $(17 / 17,100 \%)$ had dysmenorrhea and $11(11 / 17,64.7 \%)$ had menorrhagia. In non-OE group, 57 patients (57/59, 96.6\%) had dysmenorrhea and $50(50 / 59,84.7 \%)$ had menorrhagia. The improvement rates of dysmenorrhea in the two groups were $47.1 \%$ (OE group) and $86.0 \%$ (non-OE group), respectively $(\mathrm{P}<0.05)$. The improvement rates of menorrhagia in the two groups were $63.6 \%$ (OE group) and 84.0\% (non-OE group), respectively $(\mathrm{P}=0.263)$.

Conclusions: Patients without $\mathrm{OE}$ showed a lower incidence of dysmenorrhea and may have an advantage in the improvement of dysmenorrhea compared with those with OE when they underwent UAE. However, no significant difference was observed in the improvement of menorrhagia.
\end{abstract}

Keywords: Adenomyosis; dysmenorrhea; menorrhagia; ovarian endometriosis; uterine artery embolization (UAE)

Submitted Jul 08, 2020. Accepted for publication Nov 27, 2020.

doi: 10.21037/apm-20-1381

View this article at: http://dx.doi.org/10.21037/apm-20-1381

\section{Introduction}

Adenomyosis is a benign uterine disease with focal or diffuse enlargement of the uterus due to intrusion of the endometrium into the myometrium (1). The standard treatment of adenomyosis is hysterectomy, which is invasive and causes various complications (2). As the comprehensive complements to hysterectomy, hormonal treatments and endometrial ablation can temporarily induce regression of 
adenomyosis and improve the symptoms with an inadequate response (3). Currently, uterine artery embolization (UAE) has been accepted as an effective method for the treatment of adenomyosis since it is first introduced in 1995 (4). It has the advantages of minimally invasion, lower cost, preservation of the uterus and fewer complications.

Despite there are the advantages of UAE in curing adenomyosis, whether it has an equal efficacy in adenomyosis complicated with endometriosis has not been investigated yet. The clinical symptoms of adenomyosis include menorrhagia, dysmenorrhea, and dyspareunia. They are similar to those caused by endometriosis that is a benign disease characterized by the growth of endometrial tissue outside the uterus (5). Some studies have reported a significant and frequent association of uterine adenomyosis with endometriosis (6-8). The prevalence of endometriosis in adenomyosis ranges from approximately $10 \%$ to $80 \%$ (9). The most frequent location of ectopic endometrial implants outside the uterus is the ovaries (20-40\%) (10). However, the curative effect of UAE on adenomyosis with ovarian endometriosis $(\mathrm{OE})$ is rarely mentioned in the previous study.

The purpose of this retrospective study was to explore the prognostic role of ovarian endometriosis in symptomatic adenomyosis patients underwent UAE. We hypothesized that patients without $\mathrm{OE}$ showed a lower incidence of dysmenorrhea and may have an advantage in the improvement of dysmenorrhea compared with those with OE when they underwent UAE. Our study would provide a basis for UAE to treat adenomyosis with $\mathrm{OE}$. We present the following article in accordance with the STROBE reporting checklist (available at http://dx.doi.org/10.21037/ apm-20-1381).

\section{Methods}

\section{Patients}

From May 2009 to July 2016, 76 patients who underwent UAE in The First Affiliated Hospital of Sun Yat-sen University for the treatment of symptomatic adenomyosis were included in the study. This retrospective study was approved by the Institutional Review Board of The First Affiliated Hospital of Sun Yat-sen University with a waiver for informed consent [(2018)070]. The study was conducted in accordance with the Declaration of Helsinki (as revised in 2013). All patients were diagnosed as adenomyosis according to their clinical histories, symptoms, and magnetic resonance imaging (MRI) findings. The clinical histories and symptoms covered dysmenorrhea, menorrhagia, pelvic pain, and dyspareunia, among which dysmenorrhea and menorrhagia were the main complaints in this study. The diagnostic criteria for MRI of adenomyosis were as follows: (I) maximum thickness of the junctional zone $>12 \mathrm{~mm}$ and (II) high-intensity signal myometrium spot (11). All 76 patients exhibited an insufficient clinical response to pharmacotherapy (progestogens, hemostatic agents, or gonadotrophin-releasing hormone agonists) and expressed a strong desire to preserve the uterus without fertility requirements. Patients with fibroids and prior surgeries were also excluded in this study.

Patients in the OE group also met the following criteria. (I) Patients were diagnosed as OE by MRI. A definitive diagnosis was made when the lesion consisted of multiple, entirely hyperintense cysts on T1WI regardless of the signal intensity on T2WI or a cyst that was entirely hyperintense on T1WI and exhibited a hypointense signal (usually mixed with hyperintense areas) on T2WI (12). (II) The diameter of all ovarian endometriosis is $\leq 4 \mathrm{~cm}$, which is evidence for a lack of surgical indication (13). (III) Endometriosis is confined to the ovary, and patients with endometriosis in other locations (e.g., the parametria, Douglas pouch, anterior rectal wall, posterior bladder detrusor, ureters, and sigmoid colon, etc.) were excluded.

\section{Baseline data and symptom relief criteria}

Clinical information of the patients was collected retrospectively, including age, clinical symptoms, and whether symptomatic adenomyosis was accompanied by OE. The intensity of dysmenorrhea was classified according to the following VAS scores (14): mild, 0-3; moderate, 4-7; severe, $8-10$. The degree of menorrhagia was classified according to the number of sanitary pads in one menstrual cycle: normal (0-19 pads needed in one menstrual cycle); larger (20-30 pads needed); vast ( $>30$ pads needed). Followup interviews about symptom relief were conducted 12 months after UAE.

The improvements in dysmenorrhea and menorrhagia at 12 months after UAE were evaluated according to the following standards. A reduction of $50 \%$ or more in the VAS score of dysmenorrhea after UAE was defined as an improvement. A reduction of less than 50\% were regarded as no improvement. Equally, a reduction of $50 \%$ or more in the number of sanitary pads in one menstrual cycle was 
defined as improvement, and a reduction of less than $50 \%$ indicated no improvement.

\section{UAE procedure}

The preparation and embolization protocols for the patients were similar to those previously discussed in the literature (15). A 5-F Yashiro catheter (Cook, Bloomington, IN, USA) was advanced distally into the uterine artery through a femoral approach. The primary embolic agent was trisacryl gelatin microspheres (Biosphere Medical, Rockland, MA, USA), with a range of diameters that were categorized into the following groups: 500-700 $\mu \mathrm{m}$ diameter and 700-900 $\mu \mathrm{m}$ diameter. Embolization was performed until there was complete cessation of blood flow in the ascending uterine artery. All procedures in this research were conducted by the same experienced doctor with experience in UAE performance of over 10 years.

\section{MRI}

MRI (3.0-T Magnetom Trio scanner, Siemens Healthcare, Erlangen, Germany, phased-array body coil) of the pelvis was performed on all patients before UAE. All patients underwent axial and sagittal fast spin-echo T2WI [repetition time (TR)/echo time (TE): 3,440 ms/70 ms; matrix size, $384 \times 448$; section thickness, $5 \mathrm{~mm}$ ] and contrast-enhanced sagittal T1WI (TR/TE: $600 \mathrm{~ms} / 12 \mathrm{~ms}$; matrix size, $384 \times 282$; section thickness, $5 \mathrm{~mm}$ ). Contrast-enhanced MRI was performed $2 \mathrm{~min}$ after iv infusion of gadolinium (GE Healthcare, County Cork, Ireland) at a dose of $0.1 \mathrm{mmol} / \mathrm{kg}$ body weight.

\section{Statistical analysis}

SPSS version 24.0 was used for the statistical analyses. The Shapiro-Wilk test was performed on quantitative variables for normality test. As age was subjected to normal distribution $(\mathrm{P}>0.10)$, the independent sample $t$-test was used to compare the two groups. The rank sum test was used on categorical variables such as the intensity of dysmenorrhea and menorrhagia before UAE. The baseline indexes of two subgroups reached equilibrium $(\mathrm{P}>0.05)$, then Chi-square test was used to compare the efficacy of two subgroups. If the theoretical frequency of any cells is smaller than 5 (the total number of cases is bigger than 40), McNemar's chi-square test will be adopted. $\mathrm{P}<0.05$ was considered significantly different.

\section{Results}

Among the 76 patients, 17 patients were in the $\mathrm{OE}$ group and 59 patients were in the non-OE group. In the OE group, 17 patients $(17 / 17,100 \%)$ showed dysmenorrhea, and only $11(11 / 17,64.7 \%)$ had menorrhagia. In the nonOE group, 57 patients $(57 / 59,96.6 \%)$ had dysmenorrhea and $50(50 / 59,84.7 \%)$ had menorrhagia. Before UAE, the severity levels of dysmenorrhea and menorrhagia were divided into different degrees according to VAS scores and the number of sanitary pads in one menstrual cycle. There was no significant difference between the two groups among baseline data, including age, the severity levels of dysmenorrhea and menorrhagia $(\mathrm{P}<0.05)$.

All 76 patients underwent bilateral UAE, and the success rate was $100 \%$. No severe complications occurred during treatment or hospital stay. All patients completed the 12-month follow-up after UAE. In total, 57 of the 74 patients with dysmenorrhea $(77.0 \%)$ reported improvement, and 49 of the 61 patients with menorrhagia $(80.3 \%)$ reported improvement after UAE. Among the 17 patients combined with OE, 8 of $17(47.1 \%)$ with dysmenorrhea and 7 of $11(63.6 \%)$ with menorrhagia were reported improvement. In the non-OE group, 49 of 57 $(86.0 \%)$ with dysmenorrhea and 42 of 50 (84.0\%) with menorrhagia were reported improvement (Tables 1,2). The result of chi-square test showed statistically significant difference in dysmenorrhea relief $\left(c^{2}=11.050, \mathrm{P}=0.001\right)$. However, there was no significant difference in menorrhagia relief after UAE between the two groups $\left(c^{2}=1.253\right.$, $\mathrm{P}=0.263$ ) (Tables 1,2).

\section{Discussion}

Our study suggests that UAE is an effective midterm treatment for symptomatic adenomyosis. In this study, the two major symptoms, dysmenorrhea, and menorrhagia, were significantly relieved in patients with adenomyosis who underwent UAE. The overall improvement rates of dysmenorrhea and menorrhagia in 12 -month were $77.0 \%$ and $80.3 \%$, respectively, which was consistent with the previous outcomes (16-18). Present data supported that UAE was an efficient treatment for adenomyosis, particularly for those patients who were resistant to conventional therapy and wished to preserve the uterus.

Interestingly, the pain relief rate in the $\mathrm{OE}$ group was much lower than that in the non-OE group when viewed separately, which was not as expected. We speculated that OE itself may account for this expectation. Although the 
Table 1 Comparison of dysmenorrhea relief between two groups

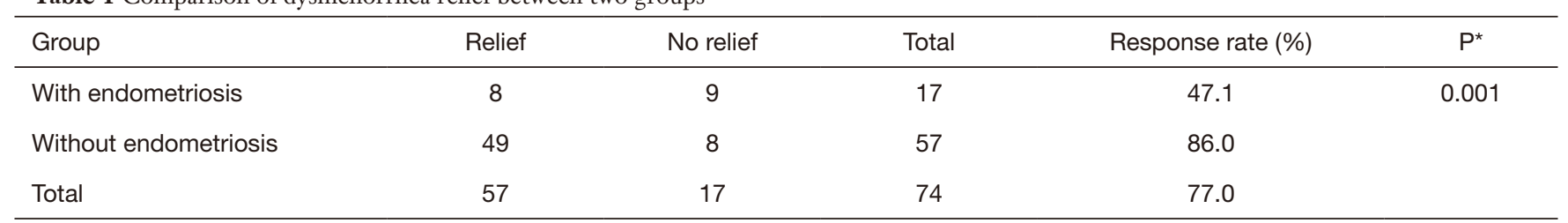

${ }^{*}$, Chi-square test was used and $\mathrm{P}<0.05$ was considered statistically significantly different.

Table 2 Comparison of menstrual relief between two groups

\begin{tabular}{lcccc}
\hline Group & Relief & No relief & Total & Response rate (\%) \\
\hline With endometriosis & 7 & 4 & 11 & 63.6 \\
Without endometriosis & 42 & 8 & 50 & 84.0 \\
Total & 49 & 12 & 61 & 80.3
\end{tabular}

*, Chi-square test was used and $\mathrm{P}<0.05$ was considered statistically significantly different.

relationship between $\mathrm{OE}$ and painful symptoms is not well elucidated and is still controversial (19), it has been reported that $38.3 \%$ of the patients with $\mathrm{OE}$ experienced pelvic pain (20). Moreover, UAE has not been previously reported to be able to cure OE. The conventional treatments of $\mathrm{OE}$ include nonsurgical therapy based on mechanisms of action (iatrogenic menopause, pseudopregnancy and antiinflammatory action) and surgery such as complete excision of the cyst wall, followed by ablation or coagulation of the cyst wall. Ovariectomy is the ultimate option (Figure 1). Therefore, OE itself may contributed to the pain in adenomyosis and UAE would do no help.

Additionally, patients with adenomyosis coexisting endometriosis may have a greater degree of uterine destruction, since endometrial glands and stroma in endometriosis are endowed with malignant biological characteristics. Endometriosis is not only able to invade the adjacent myometrium but also deeply infiltrate the distal myometrium close to the subserosa. The lesion of adenomyosis with $\mathrm{OE}$ may show poor response to UAE. Zheng reported that endometriosis was an unfavorable predictive factor for UAE, and patients combined with endometriosis were more prone to incomplete necrosis after UAE (21). However, there is no research available to explain the findings of this article, further study is needed.

Furtherly, our results showed no significant relationship in menorrhagia relief after UAE between the two groups (adenomyosis with $\mathrm{OE}$ and non-OE), which was consistent with our expectation before study. The lesion of adenomyosis increase the uterine volume and prolong the endometrium, thus leading to menorrhagia. However, $\mathrm{OE}$ has no effect on endometrium as well as menstrual quantity. UAE can cause different degrees of necrosis and reduced uterine volume, thus lead to a decrease in menstrual quantity.

Despite the favorable results, there are still several limitations in this study. First, the retrospective nature of the research itself and the single-center data limit the persuasion of the statistical analysis. Selection bias cannot be ruled out. Larger and prospective cohort studies are necessary to confirm the applicability of the results in the future. Second, the absolute value of symptoms, including the degree of dysmenorrhea and menorrhagia, is difficult to determine, which may lead to symptomatic bias. Third, the sample size is small because there are only $17 \mathrm{OE}$ cases, which causes that there is at high risk of false negative findings. Other limitations of this study are the small sample size, short follow-up time and lack of completeness in follow-up data, which may claim undurable results.

\section{Conclusions}

Taken together, UAE is an effective midterm treatment for symptomatic adenomyosis. The improvement of dysmenorrhea in the patient with adenomyosis companying OE after UAE might be less than those without endometriosis according to the mid-term outcomes of UAE. However, the improvement of menorrhagia in the patients with adenomyosis after UAE might not be affected by the accompanying $\mathrm{OE}$ or not. 

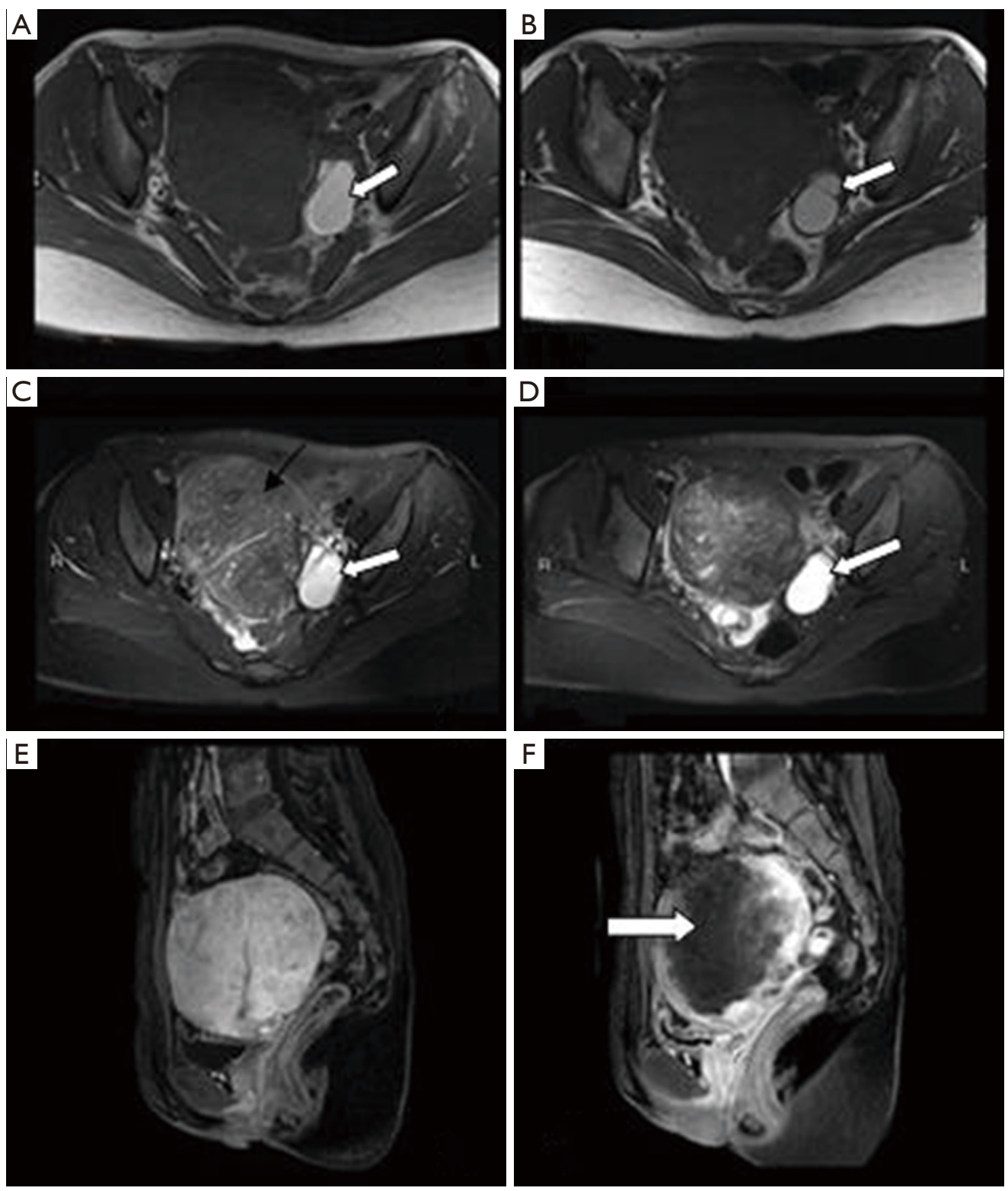

Figure 1 The MR imaging of adenomyosis patient with endometriosis. A 34-year-old women with diffuse adenomyosis accompanying endometriosis: (A) preprocedural axial T1WI with relative hyperintense (A, white arrow) and T2WI with the hyperintensity (C, white arrow) respectively show a left ovarian endometriosis; (B) postoperation axial T1WI and T2WI show the left ovarian endometriosis is still visible (white arrow); (C) preprocedural axial T2WI shows a diffuse thickening of the junctional zone accompanying punctuate T2 high signal intensity myometrial foci (black arrow); (D) postoperation axial T1WI and T2WI show the ovarian endometriosis is still visible (white arrow); (E) preprocedural sagittal contrast-enhanced T1WI shows homogenous enhancement of lesion; (F) postoperation sagittal contrastenhanced T1WI shows an irregularly shaped partial necrosis with no enhancement (white arrow). 


\section{Acknowledgments}

Funding: None.

\section{Footnote}

Reporting Checklist: The authors have completed the STROBE reporting checklist. Available at http://dx.doi. org/10.21037/apm-20-1381

Data Sharing Statement: Available at http://dx.doi. org/10.21037/apm-20-1381

Conflicts of Interest: All authors have completed the ICMJE uniform disclosure form (available at http://dx.doi. org/10.21037/apm-20-1381). The authors have no conflicts of interest to declare.

Ethical Statement: The authors are accountable for all aspects of the work in ensuring that questions related to the accuracy or integrity of any part of the work are appropriately investigated and resolved. The study was conducted in accordance with the Declaration of Helsinki (as revised in 2013). This retrospective study was approved by the Institutional Review Board of The First Affiliated Hospital of Sun Yat-sen University with a waiver for informed consent [(2018)070].

Open Access Statement: This is an Open Access article distributed in accordance with the Creative Commons Attribution-NonCommercial-NoDerivs 4.0 International License (CC BY-NC-ND 4.0), which permits the noncommercial replication and distribution of the article with the strict proviso that no changes or edits are made and the original work is properly cited (including links to both the formal publication through the relevant DOI and the license). See: https://creativecommons.org/licenses/by-nc-nd/4.0/.

\section{References}

1. Bird CC, McElin TW, Manalo-Estrella P. The elusive adenomyosis of the uterus--revisited. Am J Obstet Gynecol 1972;112:583-93.

2. Wood C. Surgical and medical treatment of adenomyosis. Hum Reprod Update 1998;4:323-36.

3. McCausland V, McCausland A. The response of adenomyosis to endometrial ablation/resection. Hum Reprod Update 1998;4:350-9.
4. Ravina JH, Herbreteau D, Ciraru-Vigneron N, et al. Arterial embolization to treat uterine myomata. Lancet 1995;346:671-2.

5. Sampson J. Intestinal adenomas of the endometrial type: their importance and their relation to ovarian hematomas of endometrial type (perforating hemorrhagic cysts of the ovary). Arch Surg 1922;5:217-80.

6. Kunz G, Beil D, Huppert P, et al. Adenomyosis in endometriosis--prevalence and impact on fertility. Evidence from magnetic resonance imaging. Hum Reprod 2005;20:2309-16.

7. Kunz G, Herbertz M, Beil D, et al. Adenomyosis as a disorder of the early and late human reproductive period. Reprod Biomed Online 2007;15:681-5.

8. Barrier BF, Malinowski MJ, Dick EJ Jr, et al. Adenomyosis in the baboon is associated with primary infertility. Fertil Steril 2004;82 Suppl 3:1091-4.

9. Emge LA. The elusive adenomyosis of the uterus. Its historical past and its present state of recognition. Am J Obstet Gynecol 1962;83:1541-63.

10. Chamié LP, Blasbalg R, Pereira RM, et al. Findings of pelvic endometriosis at transvaginal US, MR imaging, and laparoscopy. Radiographics 2011;31:E77-100.

11. Krentel H, Cezar C, Becker S, et al. From Clinical Symptoms to MR Imaging: Diagnostic Steps in Adenomyosis. Biomed Res Int 2017;2017:1514029.

12. Togashi K, Nishimura K, Kimura I, et al. Endometrial cysts: diagnosis with MR imaging. Radiology 1991;180:73-8.

13. Cooperative Group of Endometriosis, Chinese Society of Obstetrics and Gynecology, Chinese Medical Association. Guideline for the diagnosis and treatment of endometriosis. Zhonghua Fu Chan Ke Za Zhi 2015;50:161-9.

14. Institute of Medicine (US) Committee on Pain, Disability, and Chronic Illness Behavior. Measuring pain and dysfunction. In: Osterweis M, Kleinman A, Mechanic D (eds). Pain and Disability: clinical, Behavioral, and Public Policy Perspectives. Washington, DC: National Academies Press; 1987:211-31.

15. Rabinovici J, Stewart EA. New interventional techniques for adenomyosis. Best Pract Res Clin Obstet Gynaecol 2006;20:617-36.

16. Popovic M, Puchner S, Berzaczy D, et al. Uterine artery embolization for the treatment of adenomyosis: a review. J Vasc Interv Radiol 2011;22:901-9; quiz 909.

17. Kim MD, Kim YM, Kim HC, et al. Uterine artery 
embolization for symptomatic adenomyosis: a new technical development of the 1-2-3 protocol and predictive factors of MR imaging affecting outcomes. J Vasc Interv Radiol 2011;22:497-502.

18. Liang E, Brown B, Kirsop R, et al. Efficacy of uterine artery embolisation for treatment of symptomatic fibroids and adenomyosis - An interim report on an Australian experience. Aust N Z J Obstet Gynaecol 2012;52:106-12.

19. Fauconnier A, Chapron C. Endometriosis and pelvic pain: epidemiological evidence of the relationship and

Cite this article as: Guo W, Hu S, Zeng D, Yan C, Zheng R, Gao J, Wan T, Zhuang W, Yang J. The prognostic role of ovarian endometriosis in symptomatic adenomyosis patients underwent uterine artery embolization. Ann Palliat Med 2021;10(3):2577-2583. doi: 10.21037/apm-20-1381 implications. Hum Reprod Update 2005;11:595-606.

20. Khan KN, Kitajima M, Fujishita A, et al. Pelvic pain in women with ovarian endometrioma is mostly associated with coexisting peritoneal lesions. Hum Reprod 2013;28:109-18.

21. Zheng R, Zeng D, Wan TT, et al. Predisposing factors for predicting the therapeutic response of adenomyosis after uterine artery embolization: serum CA125 levels and accompanying endometriosis. Diagn Interv Radiol 2018;24:364-71. 